\title{
Numerical Simulations of Fluid Pressure in the Human Eye
}

\author{
T.R. Crowder * V.J. Ervin * \\ Department of Mathematical Sciences \\ Clemson University \\ Clemson, SC, 29634-0975 \\ USA.
}

January 22, 2013

\begin{abstract}
In this article we present computational results for the pressure in the human eye. Pressure computations for different flow rates of the aqueous humor, viscosity of the aqueous humor, and permeability of the trabecular meshwork are given. The fluid flow is assumed to be axisymmetric, and modeled as a coupled systems of Stokes and Darcy fluid flow equations, represented the fluid flow in the anterior cavity and trabecular meshwork, respectively. Rewriting the problem in cylindrical coordinates reduces the 3-D problem to a problem in 2-D. Computations are also given for varying angles between the base of the iris and the trabecular meshwork.
\end{abstract}

Key words. axisymmetric flow; Stokes flow, Darcy flow, coupled fluid flow

AMS Mathematics subject classifications. 74S05, 76D07, 76S05

\section{Introduction}

In this article we numerically investigate fluid flow through the Anterior Cavity (AC) and the Trabecular Meshwork (TM) of the human eye. Of particular interest is the dependence of the pressure difference between the $\mathrm{AC}$ and the $\mathrm{TM}$ on (i) the flow rate of the aqueous humor, (ii) the viscosity of the aqueous humor, and (iii) the permeability of the TM. Our interest in modeling the pressure in the eye is due to glaucoma, the second most common cause of blindness in the United States, which in most cases is due to increased pressure in the eye [24]. An illustration of fluid flow through the eye is given in Figures 1.1-1.2. In the eye fluid is generated by the ciliary muscle, located along side of, and behind, the lens. The fluid then flows through the AC and the TM before exiting through the Canal of Schlemm.

The model geometry of the eye used in our numerical simulations is shown in Figure 2.3. We assume that the eye is positioned looking up, and that the eye in this orientation is symmetric about the

\footnotetext{
*email: theronc@clemson.edu , vjervin@clemson.edu.
} 


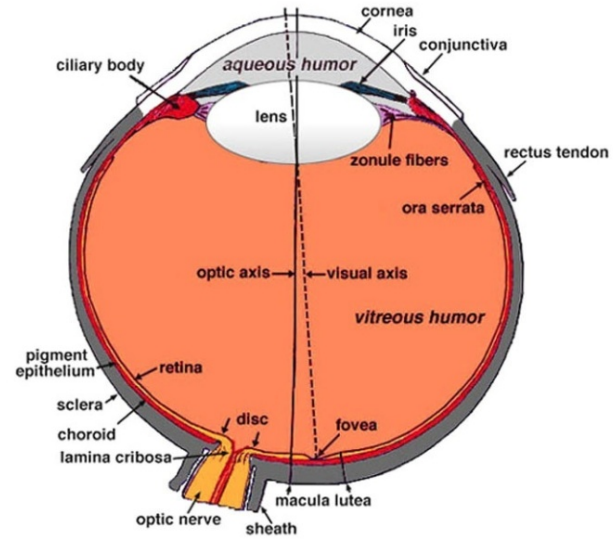

Figure 1.1: Illustration of anatomy of an eye [20].

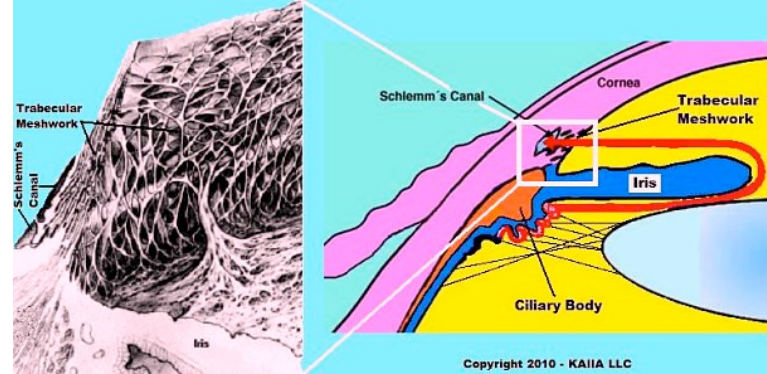

Figure 1.2: Illustration of flow through an eye $[31]$.

vertical axis. Additionally we assume that the fluid flow is axisymmetric, enabling the fluid flow (through a change of variable to cylindrical coordinates) to be modeled as a 2-D problem.

There are a number of assumptions made in the construction of any computational model. Together with the symmetry assumptions mentioned in the previous paragraph, for the computations presented we assume that there is no temperature variation across the eye, the aqueous humor is a Newtonian fluid, the flow through the TM can be model using the Darcy fluid flow equations with the model parameter (the effective fluid viscosity) dependent upon the viscosity of the aqueous humor and a permeability value for the TM. The fluid flow in the AC we model using the Stokes fluid flow equations. In [13] existence and uniqueness of the the discrete approximation to the coupled Stokes-Darcy fluid flow problem in an axisymmetric domain was established, together with a priori error estimates. Numerical computations on a model test problem demonstrated that the approximations satisfied the predicted error estimates. The computations presented below used the same numerical approximation algorithm as studied in [13].

Using the "lubrication theory" limit of the Navier-Stokes equations, together with the Boussinesq approximation for the buoyancy, buoyancy driven fluid flow in the $\mathrm{AC}$, caused by a temperature gradient across the AC, have been investigated in [7, 11, 2, 15]. Canning et al. in [7] derived a closed form solution for the buoyancy driven flow and used it to investigate the motion and distribution of particulates in the AC. El-Shahed and Abd elmaboud [11] extended the model of Canning et al. by considering a slip-flow boundary condition for the velocity along the cornea. This model was further extended by Avtar and Srivastava in [2] wherein, along with a slip-flow boundary condition for the velocity, they also assumed a convection heat conduction boundary condition on the cornea. In [15] Fitt and Gonzalez studied fluid flow in the AC caused by a temperature gradient, flow generated by the ciliary body, and flow generated by lens tremors. A model for flow fluid through the TM was considered by Avtar and Srivastava in [3]. For the TM modeled as an annular cylindrical ring, they were able to construct closed form solutions for the 1-D model (function of the radial variable $r$ ) for the pressure and the fluid velocity.

Numerical simulation in 3-D of fluid flow through the AC and TM have been performed by Kumar [21] and Kathawate [19]. For their computational models they used the Navier-Stokes equations 
with the Boussinesq approximation together, with the energy equation, for the flow in the AC and used the Darcy-Forchheimer equations for the fluid flow in the TM. They modeled the TM as being comprised of two pieces, one for the uveal and corneoscleral meshwork and the other for the juxtacanalicular tissue.

Presented in the next section is the description of the computational model used for the eye. Section 3 describes the abstract mathematical formulation for the coupled Stokes-Darcy fluid flow problem in an axisymmetric domain, together with the numerical approximation scheme. Numerical computations for the difference between the average pressure in the $\mathrm{AC}$ and the average pressure in the TM as a function of the viscosity of the aqueous humor, the permeability of the TM, and the flow rate of the aqueous humor are presented in Section 4.

\section{Computational model of the eye}

An illustration of the computational model used for the eye is given in Figure 2.3.

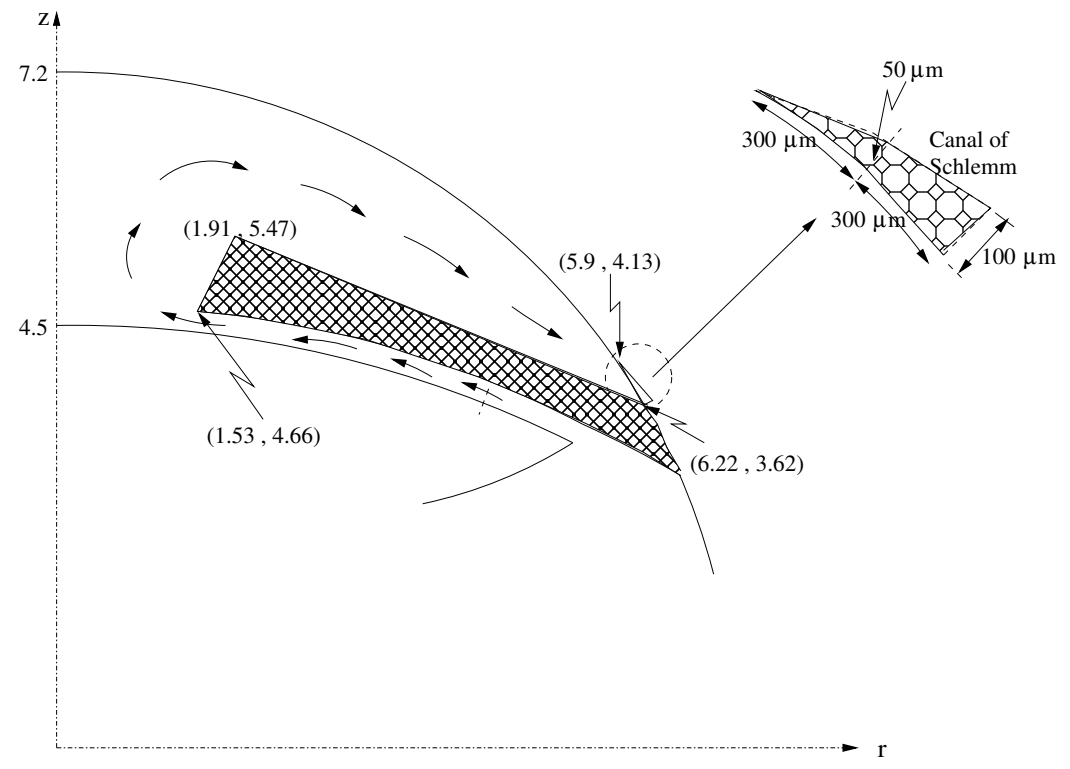

Figure 2.3: Model for simulating fluid flow through the eye.

The model geometry was constructed using $[9,10]$ and $[28]$. For the model we assume:

1. The radius of the inside of the cornea is $7.2 \mathrm{~mm}$.

2. The radius of the lens is $12.5 \mathrm{~mm}$.

3. The distance between the lens and the cornea along the vertical axis is $2.7 \mathrm{~mm}$.

4. The pupil aperture is $3 \mathrm{~mm}$. 
5. The lower side of the iris has the same curvature as the lens. The top side of the iris is approximated as a straight line. The width of the iris is approximately $0.5 \mathrm{~mm}$, and we assume the iris attaches to the cornea. (Physically the iris attaches to the ciliary muscles very near the cornea.)

6. The gap between the iris and the lens is $0.25 \mathrm{~mm}$.

7. The length of the interface between the $\mathrm{AC}$ and $\mathrm{TM}$ is $0.6 \mathrm{~mm}$.

8. The width of the TM at the bottom is $0.1 \mathrm{~mm}$.

9. The length of the interface of the TM with the Canal of Schlemm is $0.3 \mathrm{~mm}$.

10. A straight line connects the point at the top of the interface of the AC and TM with the point at the top of the interface of the TM with the Canal of Schlemm.

\section{Mathematical Formulation of Abstract Problem}

In this section we describe the abstract mathematical setting for the numerical simulation of fluid flow through the eye. We describe the setting for the case of a specified inflow and outflow flow rate, which represents a degenerate boundary condition. For a rigorous mathematical description of the formulation see [13].

Let $\breve{\Omega} \subset \mathbb{R}^{3}$, denote the flow domain of interest. Additionally, let $\breve{\Omega}_{f}$ and $\breve{\Omega}_{p}$ denote bounded convex polygonal domains for the Stokes flow and Darcy flow, respectively. The interface boundary between the domains is denoted by $\breve{\Gamma}:=\partial \breve{\Omega}_{f} \cap \partial \breve{\Omega}_{p}$. Note that $\breve{\Omega}:=\breve{\Omega}_{f} \cup \breve{\Omega}_{p} \cup \breve{\Gamma}$. The outward pointing unit normal vectors to $\breve{\Omega}_{f}$ and $\breve{\Omega}_{p}$ are denoted $\breve{\mathbf{n}}_{f}$ and $\breve{\mathbf{n}}_{p}$, respectively. On $\breve{\Gamma}$ let $\breve{\mathbf{t}}_{1}, \breve{\mathbf{t}}_{2}$ denote linearly independent unit tangent vectors. We assume that there is an inflow boundary $\breve{\Gamma}_{i n}$, a subset of $\partial \breve{\Omega}_{f} \backslash \breve{\Gamma}$, which is separated from $\breve{\Gamma}$, and an outflow boundary $\breve{\Gamma}_{\text {out }}$, a subset of $\partial \breve{\Omega}_{p} \backslash \breve{\Gamma}$, which is also separated from $\breve{\Gamma}$. See Figure 3.1 for an illustration of the domain of the problem.

Define $\breve{\Gamma}_{f}:=\partial \breve{\Omega}_{f} \backslash\left(\breve{\Gamma} \cup \breve{\Gamma}_{\text {in }}\right)$, and $\breve{\Gamma}_{p}:=\partial \breve{\Omega}_{p} \backslash\left(\breve{\Gamma} \cup \breve{\Gamma}_{\text {out }}\right)$.

We assume that the flow in the porous domain $\breve{\Omega}_{p}$ is governed by the Darcy's equation subject to incompressibility of the fluid, a specified flow rate $(\Xi)$ across $\breve{\Gamma}_{\text {out }}$, and a non-penetration condition on $\breve{\Gamma}_{p}$.

For the Stokes flow:

$$
\begin{aligned}
-\nabla \cdot\left(2 \nu \mathbf{\mathbf { d }}\left(\breve{\mathbf{u}}_{f}\right)-\breve{p}_{f} \mathbf{I}\right) & =\breve{\mathbf{f}}_{f} \quad \text { in } \breve{\Omega}_{f}, \\
\nabla \cdot \breve{\mathbf{u}}_{f} & =0 \text { in } \breve{\Omega}_{f}, \\
\int_{\breve{\Gamma}_{i n}} \breve{\mathbf{u}}_{f} \cdot \breve{\mathbf{n}}_{f} d s & =-\Xi, \\
\breve{\mathbf{u}}_{f}=\mathbf{0} & \text { on } \breve{\Gamma}_{f} .
\end{aligned}
$$

where $\breve{\mathbf{u}}_{f}=\left[\begin{array}{l}u_{f x} \\ u_{f y} \\ u_{f z}\end{array}\right]=u_{f x} \mathbf{e}_{x}+u_{f y} \mathbf{e}_{y}+u_{f z} \mathbf{e}_{z}$, for $\mathbf{e}_{x}, \mathbf{e}_{y}, \mathbf{e}_{z}$ denoting unit vectors in the $x, y$ and $z$ directions, respectively, and $\breve{\mathbf{d}}(\breve{\mathbf{u}}):=1 / 2\left(\nabla \breve{\mathbf{u}}+(\nabla \breve{\mathbf{u}})^{T}\right)$ represents the deformation tensor. 

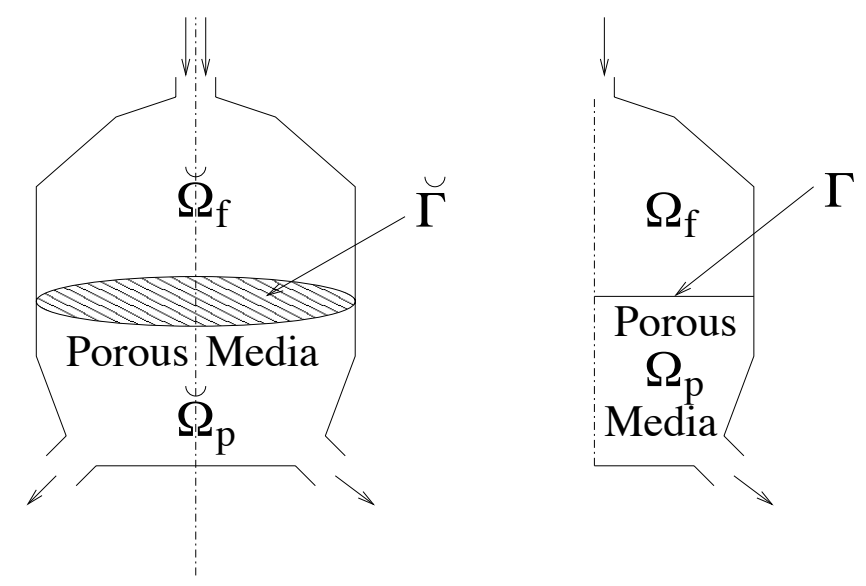

Figure 3.1: Illustration of axisymmetric flow domain.

In (3.1)-(3.4) $\breve{\mathbf{u}}_{f}$ denotes the fluid's velocity, $\breve{p}_{f}$ the pressure, $\breve{\mathbf{f}}_{f}$ an external forcing function, $\nu$ the fluid kinematic viscosity, and $\Xi$ a specified inflow rate for the fluid.

For the porous domain $\breve{\Omega}_{p}$ :

$$
\begin{aligned}
\nu_{\text {eff }} \breve{\mathbf{K}}^{-1} \breve{\mathbf{u}}_{p}+\nabla \breve{p}_{p} & =\breve{\mathbf{f}}_{p} \quad \text { in } \breve{\Omega}_{p}, \\
\nabla \cdot \breve{\mathbf{u}}_{p} & =0 \quad \text { in } \breve{\Omega}_{p}, \\
\int_{\breve{\Gamma}_{\text {out }}} \breve{\mathbf{u}}_{p} \cdot \breve{\mathbf{n}}_{p} d s & =\Xi, \\
\breve{\mathbf{u}}_{p} \cdot \breve{\mathbf{n}}_{p}=0 & \text { on } \breve{\Gamma}_{p} .
\end{aligned}
$$

In (3.5)-(3.8) $\breve{\mathbf{u}}_{p}, \breve{p}_{p}, \breve{\mathbf{f}}_{p}$, denote the fluid velocity, pressure and external forcing functions, respectively. Additionally, in (3.5) $\nu_{\text {eff }}$ represents an effective kinematic fluid viscosity, and $\breve{\mathbf{K}}$ the permeability (symmetric, positive definite) tensor of the domain. For simplicity, we let that there is a scalar function $\kappa$ such that $\kappa \mathbf{I}=\nu_{\text {eff }} \mathbf{K}^{-1}$.

Across the interface $\breve{\Gamma}$ the flows are coupled via the conservation of mass and balance of normal forces. In addition, the Beavers-Joseph-Saffman (BJS) condition $[4,18,30]$ is used for the tangential forces boundary condition on $\breve{\Gamma}$.

$$
\begin{array}{r}
\breve{\mathbf{u}}_{f} \cdot \breve{\mathbf{n}}_{f}+\breve{\mathbf{u}}_{p} \cdot \breve{\mathbf{n}}_{p}=0, \quad p_{f}-\left(2 \nu \breve{\mathbf{d}}\left(\breve{\mathbf{u}}_{f}\right) \cdot \breve{\mathbf{n}}_{f}\right) \cdot \breve{\mathbf{n}}_{f}=p_{p} \quad \text { on } \breve{\Gamma}, \\
\breve{\mathbf{u}}_{f} \cdot \breve{\mathbf{t}}_{l}=-\alpha_{l}\left(2 \nu \breve{\mathbf{d}}\left(\breve{\mathbf{u}}_{f}\right) \cdot \breve{\mathbf{n}}_{f}\right) \cdot \breve{\mathbf{t}}_{l} \quad \text { on } \breve{\Gamma}, \quad l=1,2,
\end{array}
$$

where $\alpha_{1}, \alpha_{2}$ denote friction constants.

The boundary conditions (3.3) and (3.7) are commonly referred to as defective boundary conditions, as they do not uniquely define a solution to (3.1)-(3.8). In Section 3.1 we present a weak formulation of (3.1)-(3.8) and discuss the existence and uniqueness of the weak formulation. At the end of Section 3.1 we comment that, in addition to (3.3) and (3.7), the weak formulation implicitly imposes additional boundary conditions for $\breve{\mathbf{u}}_{f}$ on $\breve{\Gamma}_{\text {in }}$ and for $\breve{\mathbf{u}}_{p}$ on $\breve{\Gamma}_{\text {out }}$. 


\subsection{Function Spaces and Weak Formulation}

In this section we introduce the function spaces needed to define the weak formulation for the coupled fluid flow problem described above.

Let $\breve{\Theta}:=\Theta \times[0,2 \pi) \subset \mathbb{R}^{3}$ be a bounded domain formed by revolving the polygon $\Theta$ around the $z$-axis. For the axisymmetric formulation we introduce the following weighted function spaces and associated norms. For any real $\alpha$ and $1 \leq p<\infty$, the space ${ }_{\alpha} L^{p}(\Theta)$ is defined as the set of measurable functions $w$ such that

$$
\|w\|_{\alpha} L^{p}(\Theta)=\left(\int_{\Theta}|w|^{p} r^{\alpha} d \mathbf{x}\right)^{1 / p}<\infty
$$

where $r=r(\mathbf{x})$ is the radial coordinate of $\mathbf{x}$, i.e. the distance of a point $\mathbf{x}$ in $\Theta$ from the symmetry axis. The subspace ${ }_{1} L_{0}^{2}(\Theta)$ of ${ }_{1} L^{2}(\Theta)$ denotes the functions $q$ with weighted integral equal to zero, $\int_{\Theta} q r d \mathbf{x}=0$.

We define the weighted Sobolev space ${ }_{1} W^{l, p}(\Theta)$ as the space of functions in ${ }_{1} L^{p}(\Theta)$ such that their partial derivatives of order less that or equal to $l$ belong to ${ }_{1} L^{p}(\Theta)$. Associated with ${ }_{1} W^{l, p}(\Theta)$ is the semi-norm $|\cdot|_{1 W^{l, p}(\Theta)}$ and norm $\|\cdot\|_{1 W^{l, p}(\Theta)}$ defined by

$$
|w|_{1 W^{l, p}(\Theta)}=\left(\sum_{k=0}^{l}\left\|\partial_{r}^{k} \partial_{z}^{l-k} w\right\|_{1 L^{p}(\Theta)}^{p}\right)^{1 / p}, \quad\|w\|_{1 W^{l, p}(\Theta)}=\left(\sum_{k=0}^{l}|w|_{1 W^{k, p}(\Theta)}^{p}\right)^{1 / p} .
$$

When $p=2$, we denote ${ }_{1} W^{l, 2}(\Theta)$ as ${ }_{1} H^{l}(\Theta)$.

For a domain $\Upsilon \subset \mathbb{R}^{3}$ we use the usual definitions and notation for Sobolev spaces. For vector functions $\mathbf{v}$ defined on $\Upsilon$ expressed in cylindrical coordinates we use the notation $\mathbf{v}(r, \theta, z)=$ $v_{r} \mathbf{e}_{r}+v_{\theta} \mathbf{e}_{\theta}+\mathbf{v}_{z} \mathbf{e}_{z}=\left(v_{r}, v_{\theta}, v_{z}\right)$, where $\mathbf{e}_{r}, \mathbf{e}_{\theta}, \mathbf{e}_{z}$ denote unit vectors in the $r, \theta$, and $z$ directions, respectively. Additionally, for $\mathbf{v}(r, z)$ defined on $\Theta$ we use $\mathbf{v}(r, z)=\left(v_{r}, v_{z}\right)=v_{r} \mathbf{e}_{r}+\mathbf{v}_{z} \mathbf{e}_{z}$.

Let $R_{\phi}$ denote a rotation with respect to $\phi$ about the $z$-axis. A function $\breve{v}$ is axisymmetric if $\breve{v}=\breve{v} \circ R_{\phi}$ for all $\phi \in(0,2 \pi)$. A vector function $\breve{\mathbf{v}}$ is rotationally invariant if $\breve{\mathbf{v}}=R_{-\phi} \circ \breve{\mathbf{v}} \circ$ $R_{\phi}$ for all $\phi \in(0,2 \pi)$. Let $\breve{H}^{s}(\breve{\Theta}) \subset H^{s}(\breve{\Theta}), s=0,1,2$ denote those functions in $H^{s}(\breve{\Theta})$ which are axisymmetric, and $\breve{H}^{s}(\breve{\Theta})^{3}$ the space of rotationally invariant $H^{s}(\breve{\Theta})^{3}$ vector functions. From $[1,5,23]$ we have the following two lemmas.

Lemma 1 For $\breve{v}(r, \theta, z) \in \breve{H}^{s}(\breve{\Theta}), s=0,1$, the mapping $\breve{v}(r, \theta, z) \rightarrow v(r, z) \in{ }_{1} H^{s}(\Theta)$ is well defined for smooth functions and (up to a factor of $\sqrt{2 \pi}$ ) is an isometry. Hence the lifting $v(r, z) \in$ ${ }_{1} H^{s}(\Theta), v(r, z) \rightarrow \breve{v}(r, \theta, z) \in \breve{H}^{s}(\breve{\Theta})$ is also an isometry (up to a factor of $\sqrt{2 \pi}$ ).

For the case $s=2$ introduce

$$
{ }_{1} H_{+}^{2}(\Theta)=\left\{v \in{ }_{1} H^{2}(\Theta): \partial_{r} v / r \in{ }_{1} L^{2}(\Theta)\right\}
$$

Lemma 2 For $\breve{v}(r, \theta, z) \in \breve{H}^{2}(\breve{\Theta})$, the mapping $\breve{v}(r, \theta, z) \rightarrow v(r, z) \in{ }_{1} H_{+}^{2}(\Theta)$ is (up to a factor of $\sqrt{2 \pi}$ ) an isometry. 
The trace space ${ }_{1} H^{1 / 2}(\Gamma)$ is defined using $\breve{H}^{1 / 2}(\breve{\Gamma})$ and the isometry between $\breve{H}^{1}(\breve{\Theta})$ and ${ }_{1} H^{1}(\Theta)$, and ${ }_{1} H^{-1 / 2}(\Gamma)$ the dual space of ${ }_{1} H^{1 / 2}(\Gamma)$.

Let $\nabla_{a}:=[\partial / \partial r, \partial / \partial z]^{T}$, and for $\mathbf{v}=\left(v_{r}, v_{z}\right), \mathbf{d}_{a}(\mathbf{v}):=1 / 2\left(\nabla_{a}(\mathbf{v})+\left(\nabla_{a}(\mathbf{v})\right)^{T}\right)$, and $\operatorname{div}_{a x i}(\mathbf{v}):=$ $\nabla_{a} \cdot \mathbf{v}+v_{r} / r$. In addition,

$$
H\left(\operatorname{div}_{a x i}, \Theta\right):=\left\{\mathbf{v}=\left(v_{r}, v_{z}\right) \in\left({ }_{1} L^{2}(\Theta)\right)^{2}: \operatorname{div}_{a x i} \mathbf{v} \in{ }_{1} L^{2}(\Theta)\right\}
$$

For $\mathbf{v} \in H\left(\operatorname{div}_{a x i}, \Theta\right),\|\mathbf{v}\|_{H\left(d i v_{a x i}, \Theta\right)}:=\left(\left\|\operatorname{div}_{a x i}(\mathbf{v})\right\|_{1 L^{2}(\Theta)}^{2}+\left\|v_{r}\right\|_{1 L^{2}(\Theta)}^{2}+\left\|v_{z}\right\|_{1 L^{2}(\Theta)}^{2}\right)^{1 / 2}$. Analogous to Lemma 2, we have the following relationship between $H\left(\operatorname{div}_{a x i}, \Theta\right)$ and $\breve{H}(\operatorname{div}, \breve{\Theta}):=\{\breve{\mathbf{v}} \in$ $\left.\breve{L}^{2}(\breve{\Theta})^{3}: \nabla \cdot \breve{\mathbf{v}} \in L^{2}(\breve{\Theta})\right\}$, where $\|\breve{\mathbf{v}}\|_{H(\operatorname{div}, \breve{\Theta})}:=\left(\|\operatorname{div}(\breve{\mathbf{v}})\|_{L^{2}(\breve{\Theta})}^{2}+\left\|v_{x}\right\|_{L^{2}(\breve{\Theta})}^{2}+\left\|v_{y}\right\|_{L^{2}(\breve{\Theta})}^{2}+\left\|v_{z}\right\|_{L^{2}(\breve{\Theta})}^{2}\right)^{1 / 2}$.

Lemma 3 For $\breve{\mathbf{v}}(r, \theta, z)=\left(\breve{v}_{r}, \breve{v}_{\theta}, \breve{v}_{z}\right) \in \breve{H}(\operatorname{div}, \breve{\Theta})$, the mapping $\left(\breve{v}_{r}, 0, \breve{v}_{z}\right) \rightarrow\left(v_{r}, v_{z}\right)=\mathbf{v}(r, z) \in$ $H\left(\right.$ div $\left._{\text {axi }}, \Theta\right)$ is (up to a factor of $\sqrt{2 \pi}$ ) an isometry.

For the description of the fluid flow in $\Omega_{f}$, we introduce the space ${ }_{1} V^{1}(\Theta)$, a subset of ${ }_{1} H^{l}(\Theta)$, given by

$$
{ }_{1} V^{1}(\Theta)=\left\{w \in{ }_{1} H^{1}(\Theta): w \in{ }_{-1} L^{2}(\Theta)\right\}, \quad \text { with norm }\|w\|_{1} V^{1}(\Theta)=\left(|w|_{1 H^{1}(\Theta)}^{2}+\|w\|_{-1}^{2} L^{2}(\Theta)\right)^{1 / 2} .
$$

The relevance of the space ${ }_{1} V^{1}(\Theta)$ is apparent from the following lemma $[1,5,23]$.

Lemma 4 For $\breve{\mathbf{v}}(r, \theta, z)=\left(\breve{v}_{r}, \breve{v}_{\theta}, \breve{v}_{z}\right) \in \breve{H}^{1}(\breve{\Theta})^{3}$, we have $\left(\breve{v}_{r}, \breve{v}_{z}\right) \rightarrow\left(v_{r}, v_{z}\right) \in{ }_{1} V^{1}(\Theta) \times{ }_{1} H^{1}(\Theta)$.

In order to incorporate the homogeneous boundary condition for the velocity on $\Gamma_{f}$, let

${ }_{1} H_{\diamond}^{1}\left(\Omega_{f}\right)=\left\{w \in{ }_{1} H^{1}\left(\Omega_{f}\right): w=0\right.$ on $\left.\Gamma_{f}\right\}, \quad$ and ${ }_{1} V_{\diamond}^{1}\left(\Omega_{f}\right)=\left\{w \in{ }_{1} V^{1}\left(\Omega_{f}\right): w=0\right.$ on $\left.\Gamma_{f}\right\}$.

For convenience of notation, let $X_{f}:={ }_{1} V_{\diamond}^{1}\left(\Omega_{f}\right) \times_{1} H_{\diamond}^{1}\left(\Omega_{f}\right),\|\mathbf{v}\|_{X_{f}}=\left(\left\|v_{r}\right\|_{1 V^{1}\left(\Omega_{f}\right)}^{2}+\left|v_{z}\right|_{1 H^{1}\left(\Omega_{f}\right)}^{2}\right)^{1 / 2}$, and $M_{f}:={ }_{1} L^{2}\left(\Omega_{f}\right)$ with $\|\cdot\|_{M_{f}}=\|\cdot\|_{1} L^{2}\left(\Omega_{f}\right)$.

The underlying space for the fluid velocity in $\Omega_{p}$ is $H\left(\operatorname{div}_{a x i}, \Omega_{p}\right)$. However a function $\mathbf{w}$ in this space may not have sufficient regularity for its trace to exist on $\partial \Omega_{p}$. Hence, the interpretation of the boundary condition $\left.\mathbf{w} \cdot \mathbf{n}\right|_{\Gamma_{p}}=0$ needs to be carefully defined. For $\breve{\mathbf{v}} \in \breve{H}\left(\operatorname{div}, \breve{\Omega}_{p}\right)$ we have that $\breve{\mathbf{v}} \cdot \breve{\mathbf{n}} \in \breve{H}^{-1 / 2}\left(\breve{\Omega}_{p}\right)$. For $\mathbf{v} \in H\left(\operatorname{div}_{a x i}, \Omega_{p}\right), \lambda \in{ }_{1} H^{1 / 2}(\Gamma)$, following Galvis and Sarkis [17] (see also [14]), we define the operator $\mathbf{v} \cdot \mathbf{n}_{p} \in{ }_{1} H^{-1 / 2}(\Gamma)$ via an extension operator $E_{\Gamma} \lambda$. Specifically, for $\breve{\lambda} \in \breve{H}^{1 / 2}(\Gamma)$ the axisymmetric lifting of $\lambda$ from $\Gamma$ to $\breve{\Gamma}$, define $\breve{E}_{\breve{\Gamma}} \breve{\lambda}:=\gamma_{0} \breve{\varphi}$, where $\gamma_{0}$ is the trace operator from $\breve{H}^{1}\left(\breve{\Omega}_{p}\right)$ to $\breve{H}^{1 / 2}\left(\partial \breve{\Omega}_{p}\right)$, and $\breve{\varphi} \in \breve{H}^{1}\left(\breve{\Omega}_{p}\right)$ is the weak solution of

$$
\begin{gathered}
-\nabla \cdot \nabla \breve{\varphi}=0, \quad \text { in } \breve{\Omega}_{p}, \\
\breve{\varphi}=\left\{\begin{array}{ll}
\breve{\lambda}, & \text { on } \breve{\Gamma} \\
0, & \text { on } \breve{\Gamma}_{\text {out }}
\end{array}, \quad \partial \breve{\varphi} / \partial \mathbf{n}_{p}=0, \text { on } \partial \breve{\Omega}_{p} \backslash\left(\breve{\Gamma} \cup \breve{\Gamma}_{\text {out }}\right) .\right.
\end{gathered}
$$


$E_{\Gamma} \lambda$ is the axisymmetric restriction of $\breve{E}_{\breve{\Gamma}} \breve{\lambda}$ to $\partial \Omega_{p}$, satisfying $\left\|E_{\Gamma} \lambda\right\|_{1 H^{1 / 2}\left(\partial \Omega_{p}\right)} \leq C\|\lambda\|_{1^{1 / 2}(\Gamma)}$. Then, we define the operator $\mathbf{v} \cdot \mathbf{n} \in{ }_{1} H^{-1 / 2}(\Gamma)$ as

$$
\langle\mathbf{v} \cdot \mathbf{n}, \lambda\rangle_{\Gamma}:=\left\langle\mathbf{v} \cdot \mathbf{n}, E_{\Gamma} \lambda\right\rangle_{\partial \Omega_{p}}=\frac{1}{2 \pi}\left\langle\breve{\mathbf{v}} \cdot \breve{\mathbf{n}}_{p}, \breve{E}_{\breve{\Gamma}} \breve{\lambda}\right\rangle_{\partial \breve{\Omega}_{p}},
$$

where $\langle\cdot, \cdot\rangle_{\partial \Omega_{p}}$ denotes the ${ }_{1} L^{2}\left(\partial \Omega_{p}\right)$ inner product, extended to a duality pairing.

For the description of the fluid flow in $\Omega_{p}$, let

$$
\begin{gathered}
X_{p}:=\left\{\mathbf{w} \in H\left(\operatorname{div}_{a x i}, \Omega_{p}\right):\left.\mathbf{w} \cdot \mathbf{n}\right|_{\Gamma_{p}}=0\right\}, \quad M_{p}:={ }_{1} L^{2}\left(\Omega_{p}\right) . \\
\text { and }\|\mathbf{w}\|_{X_{p}}:=\left(\left\|\operatorname{div}_{a x i}(\mathbf{w})\right\|_{1}^{2} L^{2}\left(\Omega_{p}\right)\right. \\
\text { a }
\end{gathered}
$$

Let

$$
X:=X_{f} \times X_{p}, \text { and } \quad M:=\left\{q \in M_{f} \times M_{p}: \int_{\Omega} q r d \mathbf{x}=0\right\},
$$

and denote the dual space of $X$ by $X^{*}$.

The axisymmetric weak formulation for (3.1)-(3.10) may be stated as: Given $\mathbf{f} \in X^{*}, \Xi \in \mathbb{R}$, determine $(\mathbf{u}, p, \lambda, \boldsymbol{\beta}) \in X \times M \times{ }_{1} H^{1 / 2}(\Gamma) \times \mathbb{R}^{2}$ such that, for all $\mathbf{v} \in X$ and $(q, \zeta, \varrho) \in M \times$ ${ }_{1} H^{1 / 2}(\Gamma) \times \mathbb{R}^{2}$,

$$
\begin{aligned}
a(\mathbf{u}, \mathbf{v})-b(\mathbf{v},(p, \boldsymbol{\beta}))+b_{I}(\mathbf{v}, \lambda) & =(\mathbf{f}, \mathbf{v}) \\
b(\mathbf{u},(q, \varrho))-b_{I}(\mathbf{u}, \zeta) & =\boldsymbol{\varrho} \cdot\left[\begin{array}{r}
-1 \\
1
\end{array}\right] \Xi /(2 \pi),
\end{aligned}
$$

where

$$
\begin{aligned}
& a(\mathbf{u}, \mathbf{v}):=a_{f}\left(\mathbf{u}_{f}, \mathbf{v}_{f}\right)+ a_{p}\left(\mathbf{u}_{p}, \mathbf{v}_{p}\right), \quad b(\mathbf{v},(q, \varrho)):=b_{f}\left(\mathbf{v}_{f},\left(q_{f}, \varrho_{1}\right)\right)+b_{p}\left(\mathbf{v}_{p},\left(q_{p}, \varrho_{2}\right)\right), \\
& b_{I}(\mathbf{v}, \zeta):=\int_{\Gamma} \mathbf{v}_{f} \cdot \mathbf{n}_{f} \zeta r d s+\left\langle\mathbf{v}_{p} \cdot \mathbf{n}_{p}, \zeta\right\rangle_{\Gamma}
\end{aligned}
$$

and

$$
\begin{aligned}
a_{f}(\mathbf{u}, \mathbf{v}) & :=\int_{\Omega_{f}} 2 \nu\left(\mathbf{d}_{a}(\mathbf{u}): \mathbf{d}_{a}(\mathbf{v})+\frac{u_{r}}{r} \frac{v_{r}}{r}\right) r d \mathbf{x}+\int_{\Gamma} \alpha_{a s}^{-1}(\mathbf{u} \cdot \mathbf{t})(\mathbf{v} \cdot \mathbf{t}) r d s, \\
a_{p}(\mathbf{u}, \mathbf{v}) & :=\int_{\Omega_{p}} \kappa \mathbf{u} \cdot \mathbf{v} r d \mathbf{x}, \\
b_{f}(\mathbf{v},(q, \beta)) & :=\int_{\Omega_{f}} q\left(\nabla_{a} \cdot \mathbf{v}+\frac{v_{r}}{r}\right) r d \mathbf{x}+\beta \int_{\Gamma_{\text {in }}} \mathbf{v} \cdot \mathbf{n}_{f} r d s, \\
b_{p}(\mathbf{v},(q, \beta)) & :=\int_{\Omega_{p}} q\left(\nabla_{a} \cdot \mathbf{v}+\frac{v_{r}}{r}\right) r d \mathbf{x}+\beta \int_{\Gamma_{\text {out }}} \mathbf{v} \cdot \mathbf{n}_{p} r d s .
\end{aligned}
$$

In (3.21) $\alpha_{a s}$ is the friction constant from the BJS condition.

\section{Equivalence of the Differential Equations and Weak Formulation}

The weak formulation (3.17)-(3.24) is obtained by multiplying the differential equations by suitably smooth functions, integrating over the domain, and using Green's theorem. Additionally, integrals 
over $\breve{\Gamma}_{\text {in }}$ and $\breve{\Gamma}_{\text {out }}$ (arising from using Green's theorem) are replaced by $\beta_{1} \int_{\breve{\Gamma}_{\text {in }}} \breve{\mathbf{v}} \cdot \breve{\mathbf{n}}_{f} d S$ and $\beta_{2} \int_{\breve{\Gamma}_{\text {out }}} \breve{\mathbf{v}}$. $\breve{\mathbf{n}}_{p} d S$, respectively. For a smooth solution the steps used in deriving the weak formulation can be reversed to show that equations (3.1)-(3.4), and (3.5)-(3.8) are satisfied. In addition, a smooth solution to (3.17)-(3.18) satisfies the following boundary conditions (see $[16,14])$.

Let $\breve{\mathbf{s}}_{t}$ on $\breve{\Gamma}_{i n}$ be given by

$$
2 \nu \breve{\mathbf{d}}(\breve{\mathbf{u}}) \breve{\mathbf{n}}_{f}=s_{n} \breve{\mathbf{n}}_{f}+\breve{\mathbf{s}}_{t},
$$

where $\breve{s}_{n}:=\left(2 \nu \breve{\mathbf{d}}(\breve{\mathbf{u}}) \breve{\mathbf{n}}_{f}\right) \cdot \breve{\mathbf{n}}_{f}$. Then, smooth solutions to (3.5)-(3.8) satisfy

$$
\begin{array}{ll}
\text { On } \breve{\Gamma}_{\text {in }}: & -\breve{p}_{f}+\breve{s}_{n}=-\beta_{1} \quad \text { and } \breve{\mathbf{s}}_{t}=\mathbf{0} . \\
\text { On } \breve{\Gamma}_{\text {out }}: & \breve{p}_{p}=-\beta_{2} .
\end{array}
$$

\section{$3.2 \quad$ Finite Element Approximation}

In this section we discuss the finite element approximation to the coupled axisymmetric StokesDarcy system (3.17),(3.18).

Let $\Omega_{j} \subset \mathbb{R}^{2}, j=f, p$, be a polygonal domain and let $\mathcal{T}_{j, h}$ be a triangulation of $\overline{\Omega_{j}}$. Thus, the computational domain is defined by

$$
\bar{\Omega}=\cup K ; \quad K \in \mathcal{T}_{f, h} \cup \mathcal{T}_{p, h}
$$

We assume that there exist constants $c_{1}, c_{2}$ such that

$$
c_{1} h \leq h_{K} \leq c_{2} \rho_{K}
$$

where $h_{K}$ is the diameter of triangle $K, \rho_{K}$ is the diameter of the greatest ball included in $K$, and $h=\max _{K \in \mathcal{T}_{f, h} \cup \mathcal{T}_{p, h}} h_{K}$.

We also assume that the triangulation on $\overline{\Omega_{p}}$ induces the partition on $\Gamma$, which we denote $\mathcal{T}_{\Gamma, h}$.
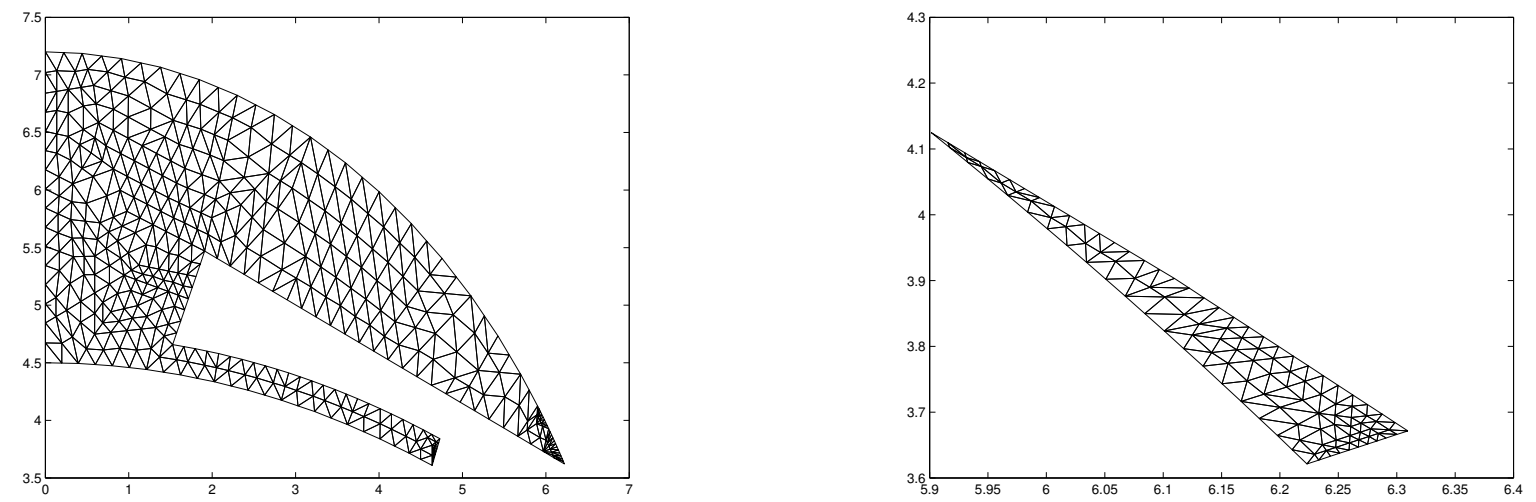

Figure 3.2: Computational mesh for the AC.

Figure 3.3: Computational mesh for the TM.

Let $P_{k}(K)$ denote the space of polynomials on $K$ of degree no greater than $k$, and $R T_{1}(K):=$ $\left(P_{1}(K)\right)^{2}+\mathbf{x} P_{1}(K)$ denote the 1st order Raviart-Thomas (R-T) elements [29,6]. Then we define 
the finite element spaces as follows.

$$
\begin{aligned}
X_{f, h}:=\left\{\mathbf{v} \in X_{f} \cap C\left(\overline{\Omega_{f}}\right)^{2}:\left.\mathbf{v}\right|_{K} \in P_{2}(K), \forall K \in \mathcal{T}_{f, h}\right\}, \\
M_{f, h}:=\left\{q \in M_{f} \cap C\left(\overline{\Omega_{f}}\right):\left.q\right|_{K} \in P_{1}(K), \forall K \in \mathcal{T}_{f, h}\right\}, \\
X_{p, h}:=\left\{\mathbf{v} \in R T_{1}(K), \forall K \in \mathcal{T}_{p, h}\right\} \\
M_{p, h}:=\left\{q \in M_{f}:\left.q\right|_{K} \in P_{1}(K), \forall K \in \mathcal{T}_{f, h}\right\} \\
L_{h}:=\left\{\zeta \in C(\Gamma):\left.\zeta\right|_{K} \in P_{1}(K), \forall K \in \mathcal{T}_{\Gamma, h}\right\} .
\end{aligned}
$$

The spaces $\left(X_{f, h}, M_{f, h}\right)$ represent the Taylor-Hood pair of approximation spaces. Analogous to the continuous formulation, we let $X_{h}:=X_{f, h} \times X_{p, h}$, and $\quad M_{h}:=\left\{q \in M_{f, h} \times M_{p, h}: \int_{\Omega} q r d \mathbf{x}=0\right\}$.

For $\left(X_{p, h}, M_{p, h}\right)$ Raviart-Thomas approximation spaces for the velocity and pressure, unlike in the Cartesian setting, $a_{p}(\cdot, \cdot)$ is not coercive, with respect to the $H\left(d i v, \Omega_{p}\right)$ norm, on

$$
Z_{p, h}:=\left\{\mathbf{v} \in X_{p, h}: \int_{\Omega_{p}} q \operatorname{div}_{a x i}(\mathbf{v}) r d \mathbf{x}=0, \forall q \in M_{p, h}\right\}
$$

To compensate for this we add the term

$$
\gamma \int_{\Omega_{p}} \operatorname{div}_{a x i}(\mathbf{u}) \operatorname{div} v_{a x i}(\mathbf{v}) r d \mathbf{x}
$$

to $a_{p}(\mathbf{u}, \mathbf{v})$, where $\gamma>0$ is a fixed constant, and define

$$
a_{p, \gamma}(\mathbf{u}, \mathbf{v}):=a_{p}(\mathbf{u}, \mathbf{v})+\gamma \int_{\Omega_{p}} \operatorname{div}_{a x i}(\mathbf{u}) \operatorname{div}_{a x i}(\mathbf{v}) r d \mathbf{x}
$$

In the approximation of Stokes and Navier-Stokes fluid flow problems in the Cartesian setting, the addition of the analogous term to (3.33) has received considerable attention recently as a means of improving the pointwise mass conservation of the approximation. (See [26, 27, 25, 22, 8].)

Discrete Approximation Problem: Given $\mathbf{f} \in X^{*}, f r \in \mathbb{R}$, determine $\left(\mathbf{u}_{h}, p_{h}, \lambda_{h}, \boldsymbol{\beta}\right) \in\left(X_{h} \times M_{h} \times\right.$

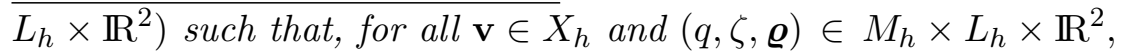

$$
\begin{aligned}
a_{\gamma}(\mathbf{u}, \mathbf{v})-b(\mathbf{v}, p, \boldsymbol{\beta})+b_{I}(\mathbf{v}, \lambda) & =(\mathbf{f}, \mathbf{v}) \\
b(\mathbf{u}, q, \varrho)-b_{I}(\mathbf{u}, \zeta) & =\boldsymbol{\varrho} \cdot\left[\begin{array}{r}
-1 \\
1
\end{array}\right] \mathrm{fr} /(2 \pi),
\end{aligned}
$$

where $a_{\gamma}(\mathbf{u}, \mathbf{v}):=a_{f}\left(\mathbf{u}_{f}, \mathbf{v}_{f}\right)+a_{p, \gamma}\left(\mathbf{u}_{p}, \mathbf{v}_{p}\right)$.

\section{Numerical Investigations}

In this section we numerically investigate the dependence of the pressure in the eye on a number of physical parameters related to fluid flow in the eye. Of specific interest is the difference between the average pressure in the $\mathrm{AC}$ and the average pressure in the TM. An increase in this pressure difference would correspond to the eye experiencing more difficulty in balancing the fluid generated in the eye with the fluid leaving the eye through the TM. 
Remark 1. The modeling equations (3.1)-(3.10) only determine the pressure up to an additive constant. Physically, this corresponds to the motion of incompressible fluids been driven by the pressure difference between two points, rather than the actual value of the pressure. Mathematically, to uniquely specify the pressure, it is convenient to impose the condition that the average pressure throughout $\Omega$ is zero. This condition was used for all the computations presented.

Remark 2. For the computation of the average pressure in the AC, AvgPreAC, we used the region of the $\mathrm{AC}$ given by the union of the triangles in Figure 3.2 lying above $z=4.66$. The average pressure in the TM, AvgPreTM, was computed using all of the triangles in the TM. The pressure difference between the average pressure in the $\mathrm{AC}$ and average pressure in the $\mathrm{TM}$ is denoted $\Delta$ Pre $=$ AvgPreAC - AvgPreTM.

Remark 3. We use the notation e- $\alpha$ to denote $10^{-\alpha}$, e.g. $3.31 \mathrm{e}-4$ denotes $3.31 \times 10^{-4}$.

\subsection{Dependence of the average pressure on the "mathematical parameter" $\gamma$}

The parameter $\gamma$ occurring in the numerical approximation is introduced to ensure that the approximating system of equations is invertible. The parameter is not part of the modeling equations. In this section with the following parameter fixed: the frictional constant, $\alpha_{B J S}=1$; kinematic viscosity in $\mathrm{AC}, \nu=0.66$; permeability of TM, $\kappa=2 \mathrm{e}-06$; effective kinematic viscosity in TM, $\nu_{e f f}=\nu / \kappa$; flow rate $=2.0 \mu \mathrm{L} / \mathrm{min}$, the dependence of the pressure difference on $\gamma$ is investigated.

Presented in Figures 4.1 - 4.4 are the velocity and pressure profiles in the AC and TM corresponding to the above parameters and $\gamma=1$.

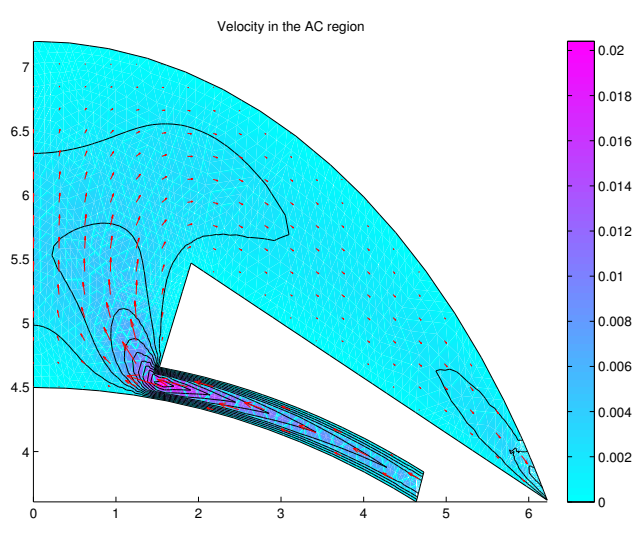

Figure 4.1: Computed flow field in the AC.

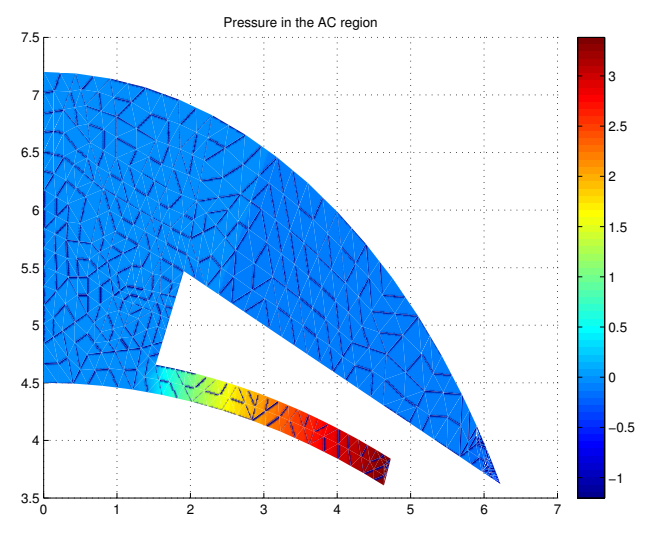

Figure 4.2: Computed pressure profile in the AC.

Remark: The mathematical parameter $\gamma$ does not appear to influence the average pressure in the $\mathrm{AC}$ and TM. For all the computations given below the value of $\gamma=1$ was used. 


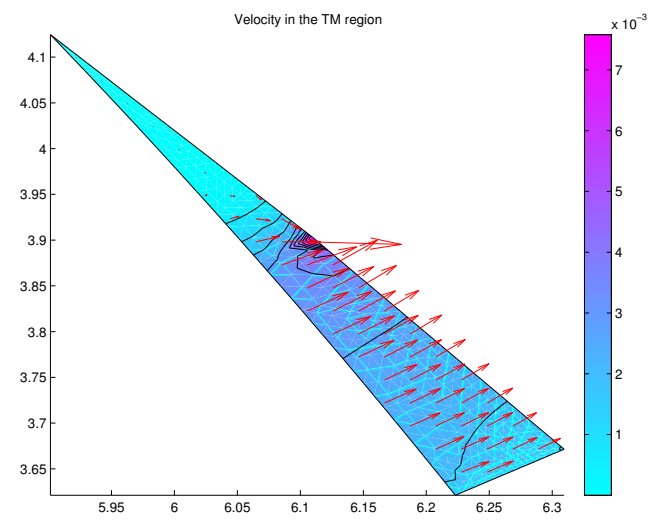

Figure 4.3: Computed flow field in the TM.

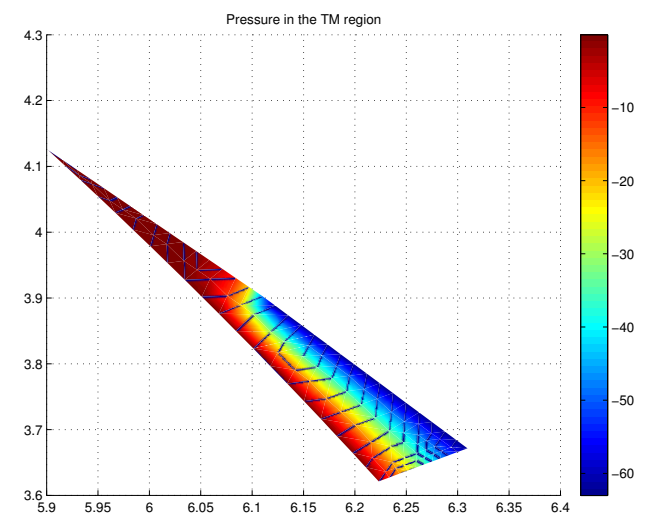

Figure 4.4: Computed pressure profile in the TM

\begin{tabular}{|c|c|c|c|}
\hline$\alpha_{B J S}=1$ & AvgPreAC & AvgPreTM & $\Delta$ Pre \\
\hline \hline$\gamma=0.01$ & $-5.7427 \mathrm{e}-02$ & $-2.4961 \mathrm{e}+01$ & $2.4904 \mathrm{e}+01$ \\
\hline$\gamma=0.1$ & $-5.7427 \mathrm{e}-02$ & $-2.4961 \mathrm{e}+01$ & $2.4904 \mathrm{e}+01$ \\
\hline$\gamma=1$ & $-5.7427 \mathrm{e}-02$ & $-2.4961 \mathrm{e}+01$ & $2.4904 \mathrm{e}+01$ \\
\hline$\gamma=10$ & $-5.7427 \mathrm{e}-02$ & $-2.4961 \mathrm{e}+01$ & $2.4904 \mathrm{e}+01$ \\
\hline$\gamma=100$ & $-5.7427 \mathrm{e}-02$ & $-2.4961 \mathrm{e}+01$ & $2.4904 \mathrm{e}+01$ \\
\hline
\end{tabular}

Table 4.1: Pressure dependence on $\gamma$.

\subsection{Dependence of the average pressure on the Beavers-Joseph-Saffman tangen- tial friction coefficient}

The Beavers-Joseph-Saffman tangential friction coefficient relates the tangential component of the normal stress vector at the AC-TM interface to the tangential velocity in the AC at that interface (see $(3.10))$.

The following table shows the average pressure in the AC and TM using parameter values: $\nu=0.66$; $\kappa=2 \mathrm{e}-06 ; \nu_{\text {eff }}=\nu / \kappa ;$ flow rate $=2.0 \mu \mathrm{L} / \mathrm{min}$.

\begin{tabular}{|c|c|c|c|}
\hline$\alpha_{B J S}$ & AvgPreAC & AvgPreTM & $\Delta$ Pre \\
\hline \hline 0.1 & $-5.7593 \mathrm{e}-02$ & $-2.4950 \mathrm{e}+01$ & $2.4892 \mathrm{e}+01$ \\
\hline 1 & $-5.7427 \mathrm{e}-02$ & $-2.4961 \mathrm{e}+01$ & $2.4904 \mathrm{e}+01$ \\
\hline 10 & $-5.6367 \mathrm{e}-02$ & $-2.5033 \mathrm{e}+01$ & $2.4977 \mathrm{e}+01$ \\
\hline
\end{tabular}

Table 4.2: Pressure dependence on $\alpha_{B J S}$.

Remark: Increasing the Beavers-Joseph-Saffman tangential frictional coefficient, $\alpha_{B J S}$, results in an increase in the difference between the average pressure in the $\mathrm{AC}$ and the average pressure in the TM. 


\subsection{Dependence of the average pressure on the viscosity of the fluid}

In this section, the dependence of the pressure on the fluid viscosity $\nu$ is investigated. For the computations given the following parameter values were used: $\alpha_{B J S}=1 ; \kappa=2 \mathrm{e}-06 ; \nu_{e f f}=\nu / \kappa$; flow rate $=2.0 \mu \mathrm{L} / \mathrm{min}$.

\begin{tabular}{|c|c|c|c|}
\hline$\nu$ & AvgPreAC & AvgPreTM & $\Delta$ Pre \\
\hline \hline 0.61 & $-5.3064 \mathrm{e}-02$ & $-2.3071 \mathrm{e}+01$ & $2.3018 \mathrm{e}+01$ \\
\hline 0.66 & $-5.7427 \mathrm{e}-02$ & $-2.4961 \mathrm{e}+01$ & $2.4904 \mathrm{e}+01$ \\
\hline 0.71 & $-6.1791 \mathrm{e}-02$ & $-2.6851 \mathrm{e}+01$ & $2.6789 \mathrm{e}+01$ \\
\hline 0.76 & $-6.6155 \mathrm{e}-02$ & $-2.8741 \mathrm{e}+01$ & $2.8675 \mathrm{e}+01$ \\
\hline 0.81 & $-7.0519 \mathrm{e}-02$ & $-3.0631 \mathrm{e}+01$ & $3.0560 \mathrm{e}+01$ \\
\hline
\end{tabular}

Table 4.3: Pressure dependence on fluid viscosity $\nu$.

Remark: The computations indicate that the difference between the average pressure in the AC and the average pressure in the TM increases as the fluid viscosity increases.

\subsection{Dependence of the average pressure on the permeability of the TM}

The permeability of the TM is represented by the parameter $\kappa$. Below are computations for the average pressures for permeability values $\kappa=2 \mathrm{e}-05, \kappa=2 \mathrm{e}-06, \kappa=2 \mathrm{e}-07, \kappa=2 \mathrm{e}-08$, and $\kappa=2 \mathrm{e}-09$.

The following parameters values were used: $\alpha_{B J S}=1 ; \nu=0.66 ; \nu_{\text {eff }}=\nu / \kappa$; flow rate $=2.0$ $\mu \mathrm{L} / \mathrm{min}$.

\begin{tabular}{|c|c|c|c|}
\hline$\kappa$ & AvgPreAC & AvgPreTM & $\Delta$ Pre \\
\hline \hline 2e-05 & $-2.1230 \mathrm{e}-01$ & $-2.9939 \mathrm{e} 00$ & $2.7816 \mathrm{e} 00$ \\
\hline 2e-06 & $-5.7427 \mathrm{e}-02$ & $-2.4961 \mathrm{e}+01$ & $2.4904 \mathrm{e}+01$ \\
\hline 2e-07 & $1.4873 \mathrm{e} 00$ & $-2.4463 \mathrm{e}+02$ & $2.4314 \mathrm{e}+02$ \\
\hline 2e-08 & $1.6932 \mathrm{e}+01$ & $-2.4410 \mathrm{e}+03$ & $2.4241 \mathrm{e}+03$ \\
\hline 2e-09 & $1.7138 \mathrm{e}+02$ & $-2.4404 \mathrm{e}+04$ & $2.4233 \mathrm{e}+04$ \\
\hline
\end{tabular}

Table 4.4: Pressure dependence on the permeability of the TM.

Remark: As the permeability of the TM decreases the computed difference between the average pressure in the $\mathrm{AC}$ increases and the average pressure in the TM increases.

Figure 4.5 is a plot of the difference between the average pressure in the $\mathrm{AC}$ and the average pressure in the TM versus the permeability of the TM. The model $\Delta$ Pre $=6 \mathrm{e}-5 \times(\text { permeability })^{-0.984}$ fits the data remarkably well, with an $R^{2}$ value of 0.99996 .

\subsection{Dependence of the average pressure on the flow rate}

Next we investigate the dependence of the pressure on the fluid rate of the aqueous humor. Computations using flow rates of $1.8 \mu \mathrm{L} / \mathrm{min}, 2.0 \mu \mathrm{L} / \mathrm{min}, 2.02 \mu \mathrm{L} / \mathrm{min}$, and $2.2 \mu \mathrm{L} /$ min were performed. 


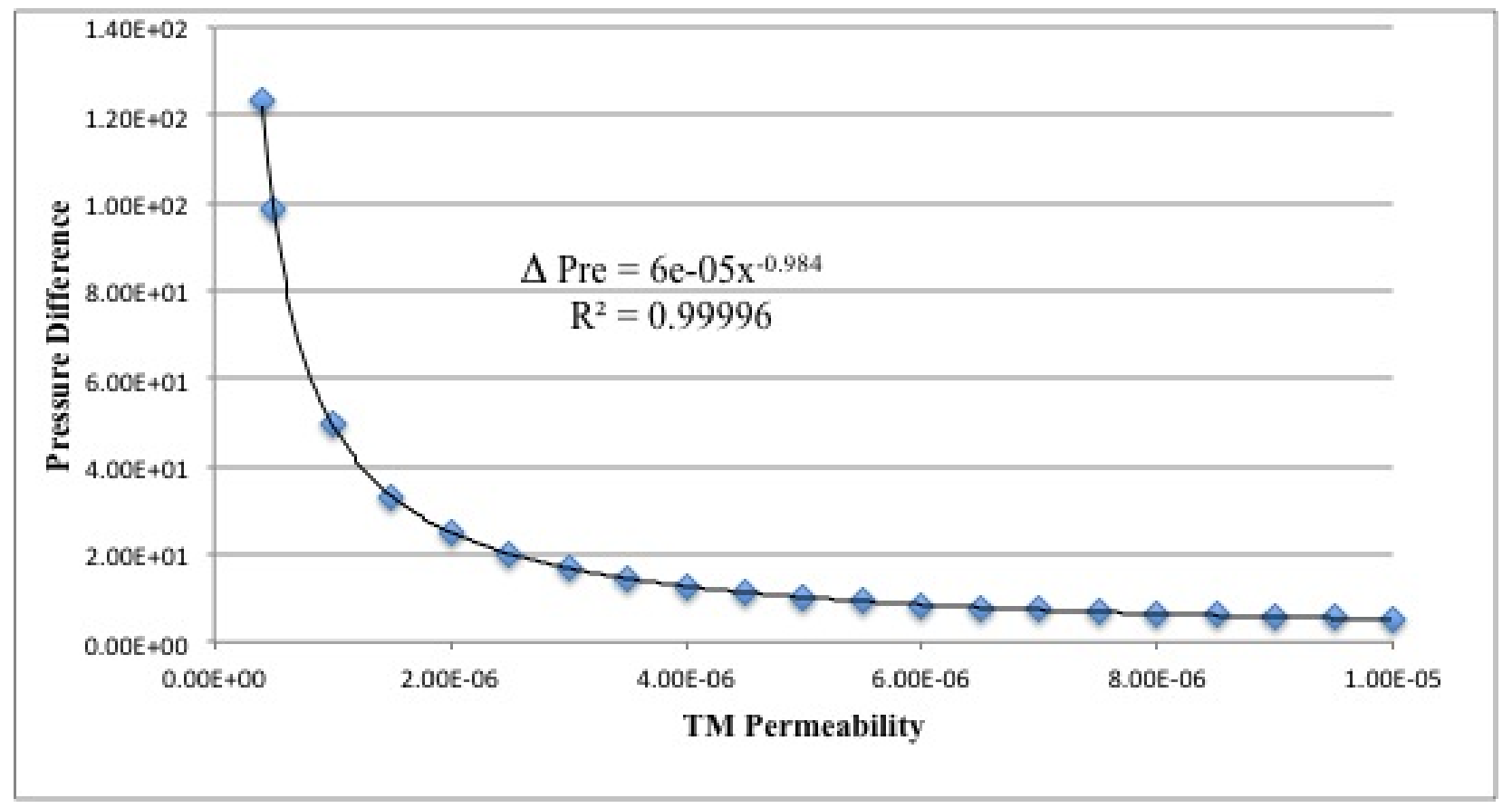

Figure 4.5: Pressure difference versus permeability of TM

The following parameters values were used: $\alpha_{B J S}=1 ; \nu=0.66 ; \kappa=2 \mathrm{e}-06 ; \nu_{e f f}=\nu / \kappa$.

\begin{tabular}{|c|c|c|c|}
\hline flow rate & AvgPreAC & AvgPreTM & $\Delta$ Pre \\
\hline \hline 1.8 & $-5.1685 \mathrm{e}-02$ & $-2.2465 \mathrm{e}+01$ & $2.2413 \mathrm{e}+01$ \\
\hline 2.0 & $-5.7427 \mathrm{e}-02$ & $-2.4961 \mathrm{e}+01$ & $2.4904 \mathrm{e}+01$ \\
\hline 2.02 & $-5.8002 \mathrm{e}-02$ & $-2.5211 \mathrm{e}+01$ & $2.5153 \mathrm{e}+01$ \\
\hline 2.2 & $-6.3170 \mathrm{e}-02$ & $-2.7457 \mathrm{e}+01$ & $2.7394 \mathrm{e}+01$ \\
\hline
\end{tabular}

Table 4.5: Pressure dependence on the flow rate.

Figure 4.6 plots the difference between the average pressure in the $\mathrm{AC}$ and the average pressure in the TM versus the flow rate. The model $\Delta$ Pre $=12.452 \times($ flowrate $)-7 \mathrm{e}-5$ fits the data remarkably well, with an $R^{2}$ value of 1.0 .

Remark: Increasing the flow rate the difference between the average pressure in the $\mathrm{AC}$ and the average pressure in the TM also increases. Indeed, a scaling argument shows that there is a linear dependence between the flow rate and the difference between the average pressure in the AC and the average pressure in the TM. 


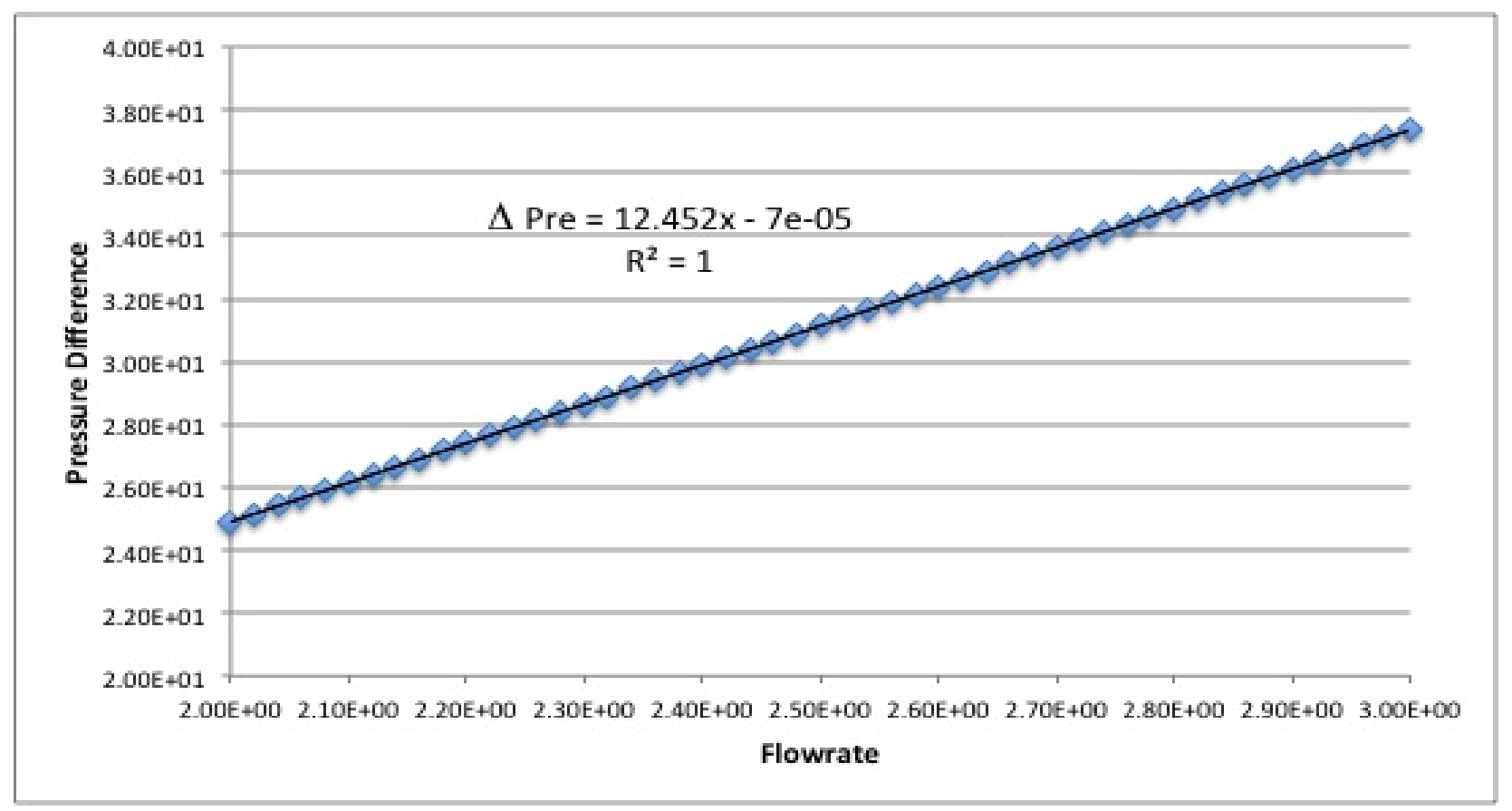

Figure 4.6: Pressure difference versus flow rate

\subsection{Dependence of the average pressure on the attachment angle of the iris to the cornea}

In this section results are given for computations performed using different angles of attachment between the iris and the cornea. All the computations given above were done with the angle of attachment $\Theta=36.59^{\circ}$. In order to vary this angle of attachment, the top of the iris was altered, while the edge closest to the lens remained unchanged. Illustrated in Figures 4.7 and 4.8 are the attachment of the iris to the cornea corresponding to angles $\Theta=36.59^{\circ}$ and $\Theta=29.73^{\circ}$.

Computations were performed with angles of attachment $\Theta=36.59^{\circ}, \Theta=34.30^{\circ}\left(93.75 \%\right.$ of $\left.36.59^{\circ}\right)$, $\Theta=32.01^{\circ}\left(87.5 \%\right.$ of $\left.36.59^{\circ}\right), \Theta=29.73^{\circ}\left(81.25 \%\right.$ of $\left.36.59^{\circ}\right)$, and $\Theta=27.4^{\circ}\left(75 \%\right.$ of $\left.36.59^{\circ}\right)$.

The following values for the model parameters were used: $\alpha_{B J S}=1 ; \nu=0.66 ; \kappa=2 \mathrm{e}-06 ; \nu_{e f f}=\nu / \kappa$; flow rate $=2.0 \mu \mathrm{L} / \mathrm{min}$.

\begin{tabular}{|c|c|c|c|}
\hline$\Theta$ & AvgPreAC & AvgPreTM & $\Delta$ Pre \\
\hline \hline $36.59^{\circ}$ & $-5.7427 \mathrm{e}-02$ & $-2.4961 \mathrm{e}+01$ & $2.4904 \mathrm{e}+01$ \\
\hline $34.30^{\circ}$ & $-5.7718 \mathrm{e}-02$ & $-2.5018 \mathrm{e}+01$ & $2.4960 \mathrm{e}+01$ \\
\hline $32.01^{\circ}$ & $-5.8319 \mathrm{e}-02$ & $-2.5090 \mathrm{e}+01$ & $2.5032 \mathrm{e}+01$ \\
\hline $29.73^{\circ}$ & $-5.7926 \mathrm{e}-02$ & $-2.5182 \mathrm{e}+01$ & $2.5124 \mathrm{e}+01$ \\
\hline $27.44^{\circ}$ & $-5.6930 \mathrm{e}-02$ & $-2.5305 \mathrm{e}+01$ & $2.5248 \mathrm{e}+01$ \\
\hline
\end{tabular}

Table 4.6: Pressure dependence on the attachment angle of the iris to the cornea.

Remark: Decreasing the angle of attachment between the iris and the cornea the difference in the 


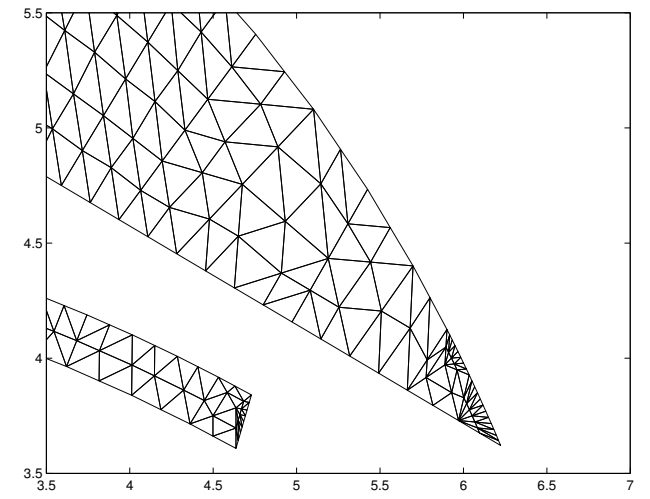

Figure 4.7: Mesh with angle of attachment between the iris and cornea $36.59^{\circ}$.

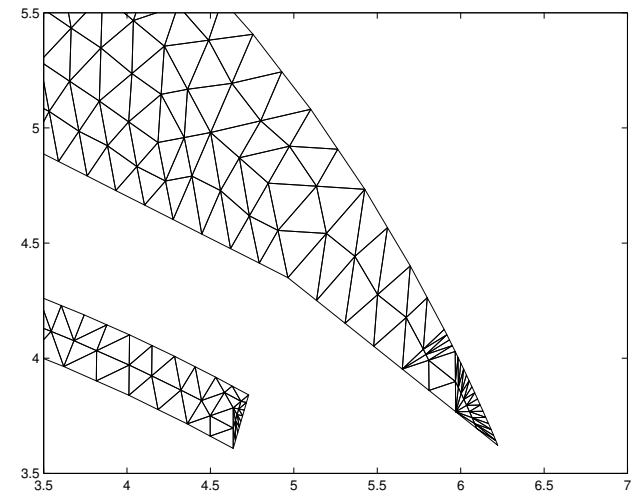

Figure 4.8: Mesh with angle of attachment between the iris and cornea $29.73^{\circ}$.

average pressure in the $\mathrm{AC}$ and the average pressure in the $\mathrm{TM}$ increases.

\section{Conclusion}

The numerical computations indicate that as the viscosity of the aqueous humor increases, or the permeability of the TM increases, or the flow rate of the aqueous humor increases, the difference between the average pressure in the $\mathrm{AC}$ and the average pressure in the TM also increases. Additionally, the numerical computations imply that the difference between the average pressure in the $\mathrm{AC}$ and the average pressure in the TM is directly proportional to (permeability of the $\mathrm{TM})^{-1}$.

\section{References}

[1] F. Assous, P. Ciarlet, Jr., and S. Labrunie. Theoretical tools to solve the axisymmetric Maxwell equations. Math. Methods Appl. Sci., 25(1):49-78, 2002.

[2] R. Avtar and R. Srivastava. Modelling the flow of aqueous humor in anterior chamber of the eye. Appl. Math. Comput., 181(2):1336-1348, 2006.

[3] R. Avtar and R. Srivastava. Modelling aqueous humor outflow through trabecular meshwork. Appl. Math. Comput., 189:734-745, 2007.

[4] G. Beavers and D. Joseph. Boundary conditions at a naturally impermeable wall. J. Fluid Mech., 30:197-207, 1967.

[5] C. Bernardi, M. Dauge, and Y. Maday. Spectral methods for axisymmetric domains, volume 3 of Series in Applied Mathematics (Paris). Gauthier-Villars, 1999. Numerical algorithms and tests due to Mejdi Azaïez. 
[6] F. Brezzi and M. Fortin. Mixed and hybrid finite element methods. Springer-Verlag, New York, 1991.

[7] C.R. Canning, M.J. Greaney, J.N. Dewynne, and A.D. Fitt. Fluid flow in anterior chamber of a human eye. IMA J. Math. Appl. Med. Biol., 19:31-60, 2002.

[8] M.A. Case, V.J. Ervin, A. Linke, and L.G. Rebholz. A connection between Scott-Vogelius and grad-div stabilized Taylor-Hood FE approximations of the Navier-Stokes equations. SIAM J. Numer. Anal., 49:1461-1481, 2011.

[9] M.W. Charles and N. Brown. Dimensions of the human eye relevant to radiation protection. Phys. Med. Biol., 20:202-218, 1975.

[10] T.S. Dietlein, P.C. Jacobi, C. Lűke, and G.K. Kreiglstein. Morphological variability of the trabecular meshwork in glaucoma patients: Implications for non-perforating glaucoma surgery. Br. J. Ophthalmol., 84:1354-1359, 2000.

[11] M. El-Shahed and Y. Abd elmaboud. On the fluid flow in the anterior chamber of a human eye with slip velocity. International Communications in Heat and Mass Transfer, 32:1104-1110, 2005 .

[12] V.J. Ervin. Approximation of axisymmetric Darcy flow. Technical report, Dept. Math. Sci., Clemson University, 2012. http://www.clemson.edu/ces/math/technical_reports/ervin.axidarcy_drv.pdf.

[13] V.J. Ervin. Approximation of coupled Stokes-Darcy flow in an axisymmetric domain. Submitted to Comp. Meth. Appl. Mech. Eng. Preprint available at http://www.ces.clemson.edu/ vjervin/papers/erv123.pdf, 2012.

[14] V.J. Ervin, E.W. Jenkins, and S. Sun. Coupled generalized non-linear Stokes flow with flow through a porous media. SIAM J. Numer. Anal., 47:929-952, 2009.

[15] A.D. Fitt and G. Gonzalez. Fluid mechanics of the human eye: Aqueous humor flow in the anterior chamber. Bull. Math. Biol., 68:53-71, 2006.

[16] L. Formaggia, J.-F. Gerbeau, F. Nobile, and A. Quateroni. Numerical treatment of defective boundary conditions for the Navier-Stokes equations. SIAM J. Numer. Anal., 40:376-401, 2002.

[17] J. Galvis and M. Sarkis. Non-matching mortar discretization analysis for the coupling StokesDarcy equations. Electron. Trans. Numer. Anal., 26:350-384, 2007.

[18] W. Jäger and A. Mikelić. On the interface boundary condition of Beavers, Joseph, and Saffman. SIAM J. Appl. Math., 60:1111-1127, 2000.

[19] J. Kathawate. Numerical solution of flow resistance in outflow pathway and intravitreal drug delivery in vitrectomised eyes. Master's thesis, Dept. Mech. Eng., Louisiana State University, December 2006.

[20] H. Kolb. Gross anatomy of the eye. at http://webvision.med.utah.edu/book/part-ifoundations/gross-anatomy-of-the-ey/. 
[21] S. Kumar. Numerical solution of ocular fluid dynamics. Master's thesis, Dept. Mech. Eng., Louisiana State University, December 2003.

[22] W. Layton, C. Manica, M. Neda, M.A. Olshanskii, and L. Rebholz. On the accuracy of the rotation form in simulations of the Navier-Stokes equations. J. Comput. Phys., 228(5):3433$3447,2009$.

[23] H. Li. Finite element analysis for the axisymmetric Laplace operator on polygonal domains. $J$. Comput. Appl. Math., 235(17):5155-5176, 2011.

[24] National Institutes of Health website. A.D.A.M. Medical Encyclopedia. at http://www.ncbi.nlm.nih.gov/pubmedhealth/PMH0002587/, 2011.

[25] M. Olshanskii, G. Lube, T. Heister, and J. Löwe. Grad-div stabilization and subgrid pressure models for the incompressible Navier-Stokes equations. Comp. Meth. Appl. Mech. Eng., 198:3975-3988, 2009.

[26] M.A. Olshanskii. A low order Galerkin finite element method for the Navier-Stokes equations of steady incompressible flow: A stabilization issue and iterative methods. Comp. Meth. Appl. Mech. Eng., 191:5515-5536, 2002.

[27] M.A. Olshanskii and A. Reusken. Grad-Div stabilization for the Stokes equations. Math. Comp., 73:1699-1718, 2004.

[28] International Commission on Radiological Protection. Task Group on Reference Man. Report of the Task Group on Reference Man. ICRP No. 23. Pergamon Press, Oxford; New York, 1975.

[29] P.-A. Raviart and J. M. Thomas. Dual finite element models for second order elliptic problems. In Energy methods in finite element analysis, pages 175-191. Wiley, Chichester, 1979.

[30] P. Saffman. On the boundary condition at the surface of a porous media. Stud. Appl. Math., 50:93-101, 1971.

[31] C. Valdivia and A. Pattatucci. Glaucoma and eye disease information. at http://www.glaucoma-eye-info.com/Open-Angle-Glaucoma.html. 